\title{
Tandem Genetic Duplications in Salmonella typhimurium: Amplification of the Histidine Operon
}

\author{
R. Philip Andersont and John R. Rotil \\ Department of Biology \\ University of $U$ tah \\ Salt Lake City, Utah 84112, U.S.A.
}

(Received 16 March 1978, and in revised form 30 June 1978)

\begin{abstract}
Salmonella strains harboring tandem duplications of chromosomal segments including the histidine operon may be selected from populations of hiso promoter-like mutants. The twofold increase in gene dosage of the histidine operon caused by tandem duplication provides resistance to concentrations of the histidine analogue 3-amino-1,2,4-triazole that inhibit the growth of haploid hisO mutants. Several properties of AT $\mathrm{T}_{+}^{+}$-resistant mutants indicate that they harbor tandem chromosomal duplications: (i) the AT-resistant phenotype of these strains is genetically unstable. Such instability is dependent upon a functional recombination system. (ii) AT-resistant mutants express approximately twice the levels of his enzymes as parental strains. (iii) Genetic tests indicate that AT-resistant mutants are merodiploid for large segments (up to $26 \%$ ) of their genome. (iv) For certain isolates, the merodiploid and AT-resistant nature of these strains are properties cotransducible with a distant chromosomal marker unrelated to the his operon. We interpret these results as indicating cotransduction of the joir-point of a tandem duplication with this distant marker.

The spontaneous frequency of tandem his duplications is remarkably high $\left(6 \cdot 2 \times 10^{-5}\right.$ per cell). This frequency is more than 6000 -fold reduced in $r e c A^{-}$ genetic backgrounds. Two major types of tanden his duplications are repeatedly isolated, having an identical $\sim 13 \%$ or $\sim 22 \%$ of their genome duplicated, respectively. When duplication-containing strains are grown under conditions that select for resistance to increased AT concentrations, clones harboring additional tandem copies (amplification) of the his operon are obtained. The role which such gene amplification may play in bacterial adaptation is discussed.
\end{abstract}

\section{Introduction}

Duplication of genetic material has been suggested to play an important role in molecular evolution (Hegeman \& Rosenberg, 1970; Ohno, 1970). Duplications may increase the dosage of a required allele (gene amplification) or supply the redundant DNA necessary for genetic divergence. Within the past several years, methods have been developed for the detection and analysis of tandem genetic duplications in bacteria and their phages (see review by Anderson \& Roth, 1977). The results of these studies suggest that tandem duplications are remarkably frequent in both Escherichia

† Present address: Medical Research Council Laboratory of Molecular Biology, Hills Road, ( ambridge CB2 2QH, England.

$\ddagger$ Abbreviation used: AT, 3-amino-1.2.4-triazole. 
coli and Salmonella typhimurium (Langridge, 1969; Miller \& Roth, 1971; Hill \& Combriato. 1973; Straus, 1974; Straus \& Hoffmann, 1975; Straus \& Straus, 1976; Anderson et al, 1976), and that such duplications may play an important role in bacterial adaptation (Novick \& Horiuchi, 1961; Horiuchi et al.. 1963: Rigby et al.. 1974; Hashimoto \& Rownd, 1975).

Two different selections for detecting tandem duplications of the histidine operon in Salmonella have previously been described. One is based upon the ability of duplication-containing strains to be made heterozygous for complementing his mutations (Anderson et al., 1976). The second is based upon the ability of tandem duplications to fuse histidine genes to "foreign" promoter elements (Anderson \& Roth, 1978). However, each of these selections suffers inadequacies that limit its usefulness for a general study of the duplication process. The selection based upon detection of complementing heterozygotes involves transduction and can only be performed in recombination-proficient $\left(\mathrm{rec}^{+}\right)$backgrounds. The selection based upon fusion of his genes to different promoter elements severely limits the chromosomal location of detectable duplication end-points.

In this paper we describe a selection for tandem duplications of the his operon that is more generally useful in studying the duplication process. It is based upon a gene dosage effect exhibited by certain his merodiploids. Strains harboring tandem duplications are selected because of their doubling of his enzyme levels. We have utilized this selection to investigate the size and frequency of spontaneously occurring tandem duplications of the his operon and the involvement of the Salmonella recombination system in this process.

\section{Materials and Methods}

(a) Media and growth conditions

Vogel \& Bonner's (1956) E medium containing $2 \%$ glucose was used as minimal medium. When required, this medium was supplemented with $0.1 \mathrm{~mm}$-histidine, 4 mM-serine, $0.4 \mathrm{~mm}$ each of adenine, guanine and arginine, $0.05 \mathrm{~mm}$-thiamin, and other amino acids (approx. $0 \cdot 3 \mathrm{~mm}$ ). DL-amino acids were often used, but the concentrations given are for the L-isomer. Adenine plus thiamin were added to all media that contained 3-amino1,2,4-triazole. Tetracycline was added to complex media at $25 \mu \mathrm{g} / \mathrm{ml}$ and to minimal media at $10 \mu \mathrm{g} / \mathrm{ml}$. When desired as a sole carbon source, D-sorbitol, D-trehalose, sodium gluconate, or glucuronic acid were added at $0 \cdot 2 \%(\mathrm{w} / \mathrm{v})$ to $\mathbf{E}$ medium from which glucose and citrate had been omitted. Difco nutrient broth $(0.8 \%)$ containing $0.5 \% \mathrm{NaCl}$ was used as complex medium. Solid media contained $1.5 \%$ Difco agar. All incubations were performed al $37^{\circ} \mathrm{C}$. Liquid cultures were aerated by gyratory shaking.

\section{(b) Bacterial strains}

The genotypes and sources of selected strains used in this study are shown in Table 1. All gene designations are those of the revised Salmonella genetic nomenclature (Sanderson \& Hartman, 1978). Strains with "TT" designations are those in our collection that either contain or are descended from a strain containing a copy of the translocatable tetracyclineresistance determinant $\operatorname{Tn} 10$ (Kleckner et al., 1975). All strains are derivatives of S. typhimurium strain LT2.

Strain TR4179 ( from strain TR3578 into strain TR4178 (his 4203 srl-201) (Anderson \& Roth, 1978), selecting $\mathrm{His}^{+}$. The mutation $\mathrm{srl}-201$ is approximately $50 \%$ linked to $\mathrm{rec} A 1$ by bacteriophage P22-mediated generalized transduction. The close proximity of these 2 loci in $E$. coli has been described (McEntee, 1976; A. J. Clark, personal communication). Strain TR4255 (hisO2355 srl-201 $\operatorname{rec} A 1$ strA) was derived by mating donor strain TR2246 
Table 1

List of strains

\begin{tabular}{|c|c|c|}
\hline Strain & Genutype & Source \\
\hline 'TR3578 & hisO2355 & P. E. Hartman \\
\hline TR4179 & hisO2355 orl-201 & This paper \\
\hline TR4255 & hisO2355 srl-201 recA I strA & 'This paper \\
\hline TR2951 & his $\triangle 63 \operatorname{recA} 1$ strA & Anderson \& Roth (1978) \\
\hline TR5368 & $\mathrm{hisO}_{2355 \mathrm{grl}^{+} \mathrm{recA}}^{+}$ & TR2951 $\times$TR4179 \\
\hline 'TR5369 & hisO2355 $\mathrm{srl}^{+} \mathrm{recAl}$ & TR2951 $\times$ TR4179 \\
\hline TT2279 & $\begin{array}{l}\text { his } \Delta 515\left(\mathrm{gnd}^{-} r f b^{-}\right) \text {met-52l eda.100/ } \mathrm{F}_{400}^{\prime} \\
\text { hisO2355 } \mathrm{gnd}^{+} r f b^{+} z z f-20:: \operatorname{Tn} 10\end{array}$ & This paper \\
\hline TT2280 & hisO2355 recA $1 / \mathbf{F}_{400}^{\prime}$ hisO2355 zaf-20:: $\operatorname{Tn} 10$ & TT2279 $\times$ TR5369 \\
\hline TT1984 & hisG8575::Tn10 hisO2355 srl-201 & 'This paper' \\
\hline TT1985 & hisD8578:: Tn 10 hisO2355 srl-201 & This paper \\
\hline TT1986 & hisC8579::Tn10 hisO2355 srl-201 & This paper \\
\hline TT1987 & hisB8686::Tn10 hisO2355 srl-20I & 'This paper \\
\hline TT1988 & hisH $8576:: \operatorname{Tn} 10$ hisO2355 srl-201 & This paper \\
\hline TT1989 & hisA $8676:: \operatorname{Tn} 10$ hisO2355 srl-201 & This paper \\
\hline TT1990 & hisF $8539:: \operatorname{Tn} 10$ hisO2355 srl.201 & This paper \\
\hline TT1991 & hisI $9537:: \operatorname{Tn} 10$ hisO23.55 arl-201 & This paper \\
\hline TT14 & $\operatorname{met} C 1975:: \operatorname{Tn} 10$ & Anderson \& Roth (1978) \\
\hline $\mathrm{TT} 126$ & $\operatorname{tyr} A 555:: \operatorname{Tn} 10$ & Anderson \& Roth (1978) \\
\hline TT142 & $\arg (x 1828:: \ln 10$ & Anderson \& Roth (1978) \\
\hline TTl46 & $\arg A 1832:: \operatorname{Tn} 10$ & Anderson \& Roth (1978) \\
\hline TT169 & $\operatorname{ser} A 977:: \operatorname{Tn} 10$ & Anderion \& Roth (1978) \\
\hline TT173 & cysC1511:: $\operatorname{Tn} 10$ & Anderson \& Roth (1978) \\
\hline TT215 & $\operatorname{lys} A 565:: \operatorname{Tn} 10$ & Anderson \& Roth (1978) \\
\hline $\mathrm{T"T} 278$ & guaA554::Tn 10 & Anderson \& Roth (1978) \\
\hline TT287 & purC882: :Tn10 & Anderson \& Roth (1978) \\
\hline 'IT315 & purG1739:: $\operatorname{Tn} 10$ & Anderson \& Roth (1978) \\
\hline TT317 & purF $1741:$ : $\operatorname{Tn} 10$ & Anderson \& Roth (1978) \\
\hline $\mathrm{TT} 418$ & glyA $540:: \operatorname{Tn} 10$ & Anderion \& Roth (1978) \\
\hline $\mathrm{TT} 11$ & purl1757::Tn10 & 'This paper' \\
\hline TT28 & hisC8579::Tn10 & This paper \\
\hline TT98 & $\operatorname{trp}-1013:: \operatorname{Tn} 10$ & This paper \\
\hline $\mathrm{TT} 464$ & pyrF696: : $\operatorname{Tn} 10$ & 'This paper \\
\hline TT520 & srl-202: : Tn 10 & This paper \\
\hline TT1339 & pheA554: : $\operatorname{Tn} 10$ & 'This paper \\
\hline TT1454 & aroD553:: $\operatorname{Tn} 10$ & This paper \\
\hline TT1518 & tre $-57:: \operatorname{Tn} 10$ & This paper \\
\hline NK 186 & cysA $1539:: \operatorname{Tn} 10$ & N. Kleckner \\
\hline TT2106 & hisH9528: : Tn5 & This paper \\
\hline $\mathrm{TT} 2420$ & hisO2355 srl-201 lysA565:: $\operatorname{Tn} 10$ & TT215 TR4179 \\
\hline
\end{tabular}

All strains are derivatives of $S$. typhimurium strain L'T2. See Materials and Methods for the derivation of strains original to this paper.

(met $A 22 \operatorname{rec} A 1 \operatorname{str} A$ HFr $B 2)$ with recipient strain TR4179. Streptomycin-resistant $\left(\mathrm{Met}^{+}\right)$ clones were selected and the desired recombinant was identified among the progeny. Strains TR5368 (hisO2355) and TR5369 (hisO2355 recA1) were obtained as Srl ${ }^{+}$transductants from a cross between donor strain TR295I (his 463 recA1 strA) and recipient strain TR4179 (hisO2355 srl-201). They are therefore isogenic, except for $\operatorname{rec} A$.

Strain TT2279 [his 4515 (gnd $\left.^{-} r f b^{-}\right)$eda-100 met-521/ $\mathrm{F}_{400}^{\prime} h i s O 2355$ gnd $\left.^{+} r f b^{+} z z f-20:: \operatorname{Tn} 10\right]$ was constructed in a series of steps from strain TR3629 [his 4515(gnd $\left.{ }^{-} \mathrm{rb}^{-}\right)$met-521]. An $e d a^{-}$mutation was introduced into strain TR3629 according to the general procedures of Peyru \& Frankel (1968). The deletion mutation his 4515 harbored by TR3629 extends well beyond the his region, removing also the genes gnd (encoding gluconate-6.phosphate 
dehydrogenase) and $r f b$. Diethylsulfate mutagenesis of strain TR3629 followed by 2 cycles of penicillin enrichment for Glk- (gluconate non-utilizing) clones yielded strain TR3949 [his $\Delta 515$ (gnd $\left.{ }^{-} \mathrm{rfb}^{-}\right)$met-521 eda-100]. It was further characterized as being unable to utilize? either D-gluconic or D-glucuronic acid as a sole carbon source. Both mutagenesis and penicillin enrichment were performed acoording to the procedures of Roth (1970). Strain TR395 1 was then constructed by mating recipient st rain TR3949 with donor strain TR4520

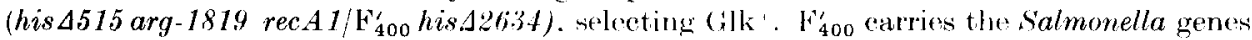
for his, gnd and $r f b$ (Voll, 1972). To prevent reeombination between this Salmonella episome and the chromosome, it is necessary that the chromosome either be rect or harbor deletion mutation his $\Delta 515$. This deletion is very large and apparently removes all chromosomal sequences carried by $\mathbf{F}_{400}^{\prime} . \mathrm{F}_{4 \text { in }}^{\prime}$ is therefore stably maintained over the chromosome of TR3949, and the resulting merogenote (TR3951) is phenotypically His ${ }^{-} \mathrm{Glk}^{+} \mathrm{Rfb}^{+}$. Strain TR3951 was then used as a transductional recipient for donor strain TR3578 (hisO2355), selecting His ${ }^{+}$. The large size of deletion his $\Delta 515$ prevents chromosomal His ${ }^{+}$ recombinants; one episomal recombinant is strain 'IR4190 [his $\Delta 515$ (gnd $\mathrm{gfb}^{-}$) eda-10)! met $-521 / \mathrm{F}_{400}^{\prime}$ hisO2355 gnd $\left.^{+} r f b^{+}\right]$. Finally, recipient strain $\mathrm{TR} 4190$ was transduced with

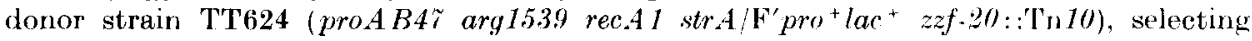
tetracycline resistance. Donor strain TT624 harbor's an insertion of the Tn10 element (isolated by $\mathrm{F}$. Chumley) into $\mathrm{F}$-sequences of an $E$. coli $\mathrm{F}^{\prime} p r o+l a \mathrm{c}^{+}$episome. This Tn 10 insertion may be transduced onto any $\mathbf{F}^{\prime}$ element, thus providing a selectable marker for' subsequent $\mathrm{F}^{\prime}$ transfor (totracycline rosistance). One such Tot $^{\mathrm{R}}$ recombinant is strain

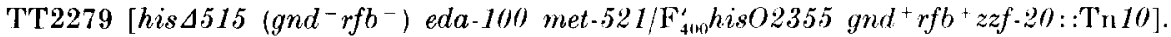

A large number of auxotrophic and fermentation-defective mutants resulting from insertion of the Tn10) element have been isolated in our laboratory as a co-operative effort. The sites of insertion have been identified in many of these mutants by as many as 3 independent tests: (i) the ability of selected biosynthetic intermediates to fulfill nutritional requirements (crystal tests); (ii) a demonstration of transductional linkage of $\operatorname{Tn} 70$ insertions to known genetic markers: and (iii) complementation tests between Tn 10 insertions and $\mathrm{F}^{\prime}$ episomes of known genotype. The results of these test have led to the unambiguous assignment of many Th 10 insertions to defined genes. Th 10 insertions into 58 such genes have thus far been identified. A small number of auxotrophs resulting from insertion of the translocatable kanamycin-resistance determinant $T n 5$ (Berg et al., 1975) have similarly been isolated and identified. Insertion mutations of interest to this study are shown in Table 1. Strains TT 1984 to 1991 were constructed by transducing his ::Tn 70 insertions into strain TR4179, selecting tetracycline resistance. The desired recombinants (hisO2355 his ${ }^{-}:$Tn 10 srl-201) were identified among the progeny as being unable to donate his ${ }^{+}$by transduetion.

\section{(c) Genetic techniques:}

\section{(i) Isolation of amino-triazole-resistant mutants}

In order to measure the frequency of AT $\dagger$-resistant mutants, nutrient broth-grown cultures of TR5368 (hisO2355) or TR5369 (hisO2355 recA1) were diluted, and samples were spread onto minimal medium containing adenine, thiamin, and $1 \cdot 8$ mm-anninotriazole. Following 2 days incubation, the number of AT-resistant clones was counted.

For the isolation and analysis of independent AT-resistant mutants, individual nutrient broth cultures were inoculated with single colonies of either strain TR4179 (hisO2355 srl-201) or TR4255 (hisO2355 srl-201 recA1 strA). Following overnight growth, samples were spread individually onto minimal medium containing adenine, thiamin, and 1.8 mM-amino-triazole. From each culture, one AT-resistant mutant was picked, purified, and further analyzed.

\section{(ii) Duplication mapping}

The mapping data presented in Tables 3 and 4 was obtained by a spot-test method. A P22 transducing lysate grown on a particular Tn10 auxotroph was spread on each of 2 minimal plates containing tetracycline $(10 \mu \mathrm{g} / \mathrm{ml})$. One of the plates also contained the

$\dagger$ See footnote to p. 53 . 
TABLe 2

hisD enzyme levels in haploid and amino-triazole-resistant strains

\begin{tabular}{|c|c|c|c|c|}
\hline Strain & Genotype & Phenotype & Ploidy & $\begin{array}{c}\text { HoHase specific } \\
\text { activity }\end{array}$ \\
\hline L'1'2 & wild-type & $A^{\prime} I^{{ }^{K}}$ & haplosd & $16 \cdot 30$ \\
\hline TR4179 & hisO235.5 & $\mathrm{AT}^{\mathrm{s}}$ & haploid & 0.40 \\
\hline TR540I & hisO2355/hisO2355 & $\mathrm{AT}^{\mathrm{T}} \mathrm{R}$ & diploid & 0.67 \\
\hline TR5402 & hisO2355/hisO2355 & $\Delta \mathrm{T}^{\mathrm{R}}$ & diploid & 0.67 \\
\hline TR5536 & hisO2355 & $\mathrm{AT}^{\mathrm{s}}$ segregant of TR5401 & haploid & 0.42 \\
\hline TR55:37 & hisO2355 & $A^{\prime} T^{\mathrm{S}}$ segregant of TR $540^{2}$ & haploid & 0.46 \\
\hline
\end{tabular}

Histidinol dehydrogenase was assayed by the procedures of Ciesla et al. (1975). Cells were grown in minimal medium plus $0.1 \mathrm{~mm}$-histidine. A his D deletion mutant shows $<0.01$ unit of activity in this assay. Figures are averages of 3 or more determinations.

$+\mathrm{AT}^{\mathrm{S}}$ and $A \mathrm{~T}^{\mathrm{K}}$ indicate sensitivity and resistance to media contamng $1.8 \mathrm{~mm}-3$-amino-1,2,4triazole, respectively.

\$ Histidinol dehydrogenase (HDHase) specific activity is expressed as (cts/min conversion of $1^{14}$ C]histidinol to $\left[{ }^{14} \mathrm{C}\right]$ histidine $/ 30 \mathrm{~min}$ per $0 . \mathrm{D}_{650}$ eells $) \times 10^{-4}$

nutritional requirements of the donor Tn10 auxotroph. Strains carrying duplications to be mapped were spotted onto these plates $\left(10^{8}\right.$ cells of each of 20 strains). Under these conditions, selection is made for Tet ${ }^{R}$ with or without a concomitant selection for prototrophy. If a given recipient yields approximately equal numbers of transductants on the 2 media $(+10 \%)$ the duplication is judged to include the site of the Tn 10 insertion. If the minimal plate yielded few recombinants ( 1 to $5 \%$ of the number seen on the supplemented plates) then it was concluded that the site of the Tn 10 insertion lies outside the duplicated segment. In most cases if Tn10 was thought to be within a duplication, several prototrophic. Tet ${ }^{\mathrm{R}}$ transductants were purified and shown to vield segregants of the 2 parental types (Tet ${ }^{\mathrm{R}}$, auxotrophic or $\mathrm{Tet}^{\mathrm{S}}$, prototrophic).

\section{(iii) Other genetic techniques}

The techniques of transduction, $\mathrm{F}^{\prime}$ transfer, and preservation of unstable strains have been previously described (Roth. 1970; Anderson \& Roth, 1978). A non-lysogenizing derivative of the high-transducing phage of Schmieger (1971). P22 HT105/1 int-201, was used in all transductions.

\section{(d) Biochemical techniques}

Radiometric assay of histidinol dehydrogenase (EC 1.1.1.23) was performed according to the procedures of Ciesla et al (1975).

\section{Results}

\section{(a) Isolation of strains having tandem duplications}

We sought to develop conditions which inhibit the growth of haploid strains but allow the growth of strains merodiploid for the his operon. These conditions permit direct selection of strains carrying tandem duplications of the histidine operon since the gene dosage increase afforded by an extra copy of the his operon is essential for growth. We have developed such conditions by challenging hisO "promoter-like" mutants with the histidine analogue 3-amino-1,2,4-triazole, an inhibitor of histidine biosynthesis. Promoter-like hisO mutations are a class of cis-dominant regulatory mutations located at one end of the operon: they cause reduced (but regulated) levels 


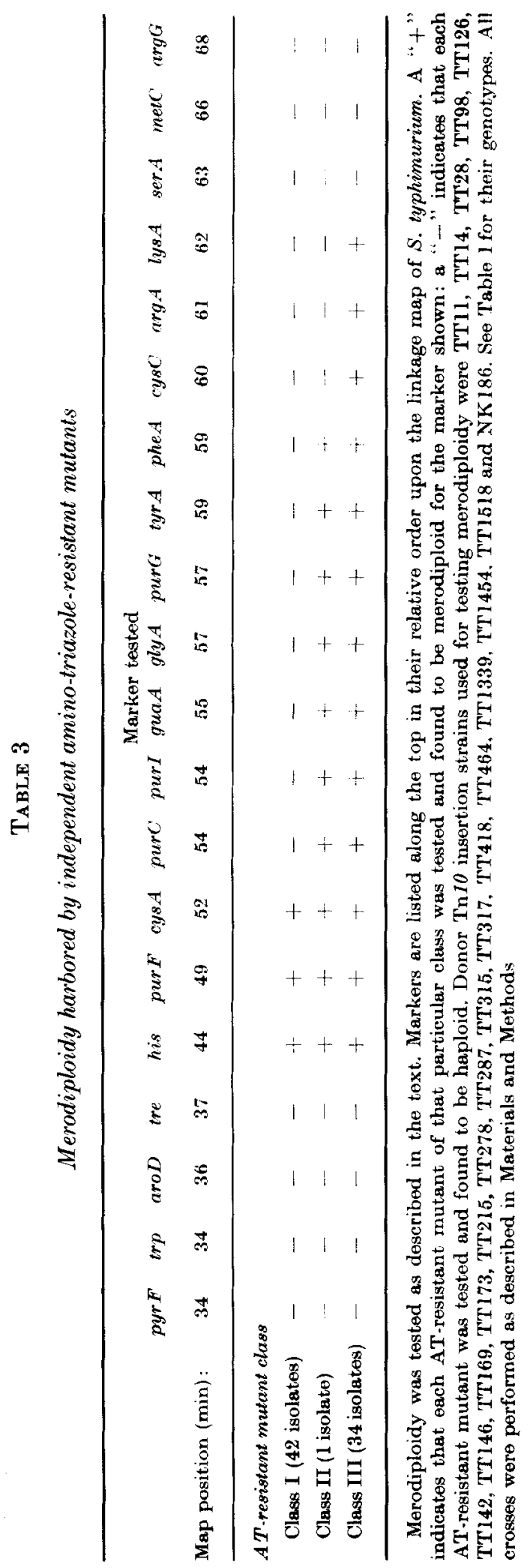




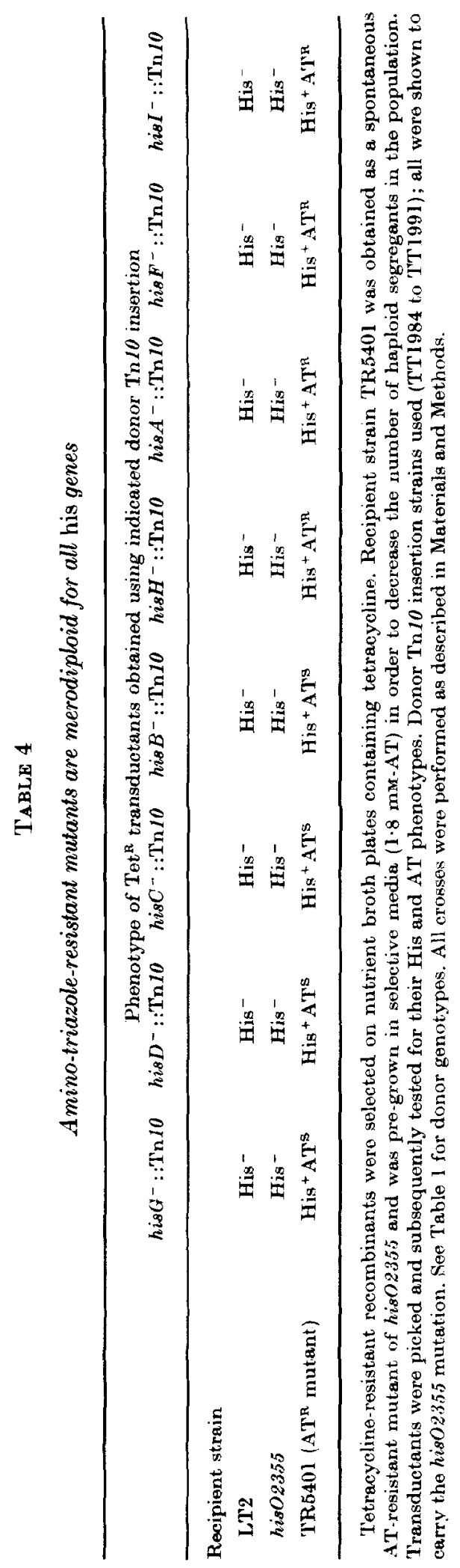


'Table 5

Cotransduction of class III amino-triazole-resistance with lys A

\begin{tabular}{|c|c|c|c|}
\hline & \multicolumn{3}{|c|}{$\begin{array}{l}\text { Recombinants in cross with } \\
\text { recipient TT2420 (hisO2355 lys A565: Tn 10) }\end{array}$} \\
\hline & No. Lys $+A^{\prime} \mathrm{I}^{\mathrm{s}}$ & No. Lys ${ }^{+} A \mathrm{I}^{\mathrm{R}}$ & $\%$ Cotransduction \\
\hline TR5435 (class III AT ${ }^{\mathrm{R}}$ ) & $82: 3$ & 17 & $2 \cdot 0$ \\
\hline TR5464 (class I AT ${ }^{\mathrm{R}}$ ) & 1304 & 0 & 0.08 \\
\hline LT2 (wild-type) & 420 & 0 & $<0 \cdot 3$ \\
\hline
\end{tabular}

$\mathrm{Lys}^{+}$recombinants were seleoted on minimal medium plus histidine plates. Transduetants were picked and subsequently tested for their AT phenotype. All crosses were performed as described in Materials and Methods.

of his enzyme expression (Ely et al., 1974). They have the properties of classical "promoter" mutations (Jacob et al., 1964; Scaife \& Beckwith, 1966). Most strains containing promoter-like his $O$ mutations exhibit sufficient his enzyme expression to be phenotypically $\mathrm{His}^{+}$. However, because of their limited ability to express the operon, such mutants are quite sensitive to growth inhibition by the histidine analogue 3-amino-1,2,4-triazole. AT is a specific inhibitor of the imidazole glycerol phosphate dehydratase activity of the his $B$ bifunctional enzyme (Hilton et al., 1965). Wild-type strains are resistant to the growth-inhibitory properties of this compound because of their ability to derepress hisB expression. Promoter-like his $O$ mutants are unable to derepress fully and therefore are sensitive to AT inhibition. At appropriate concentrations of AT, hisO promoter mutants give rise to unstable AT-resistant clones that prove to contain tandem duplications of chromosomal segments that include the his operon. The appropriate concentration of AT is the minimum necessary to inhibit growth of the his $O$ mutants. Under these conditions, the doubling of hisB activity achieved by the gene dosage effect of a tandem duplication is apparently sufficient to allow escape from AT inhibition. The minimum inhibitory AT concentration is different for each hisO mutant tested. Such differences probably reflect the maximum enzyme expression possible for various his $O$ mutants.

Growth of mutant hisO2355 (Voll, 1967) is inhibited by concentrations of AT equal to or in excess of $1.8 \mathrm{~mm}$. By comparison. growth of wild-type Salmonella is resistant to inhibition by AT concentrations above $40 \mathrm{~mm}$. When plated on media containing $1.8 \mathrm{~mm}-\mathrm{AT}$, strain hisO2355 gives rise to AT-resistant clones that are genetically unstable; that is, when grown non-selectively (in the presence of excess histidine), cultures of such mutants accumulate AT-sensitive progeny at high frequency (10 to $40 \%$ AT-sensitive segregants following 15 to 20 generations of non-selective growth). These AT-sensitive clones are identical in all respects to the parental mutant his 02355 .

The instability of AT-resistant clones is demonstrated in Figure 1. A single colony of an AT-resistant mutant obtained from his02355 was inoculated into nutrient broth and grown for 10 to 15 generations. This culture was diluted and samples were spread onto plates containing $1.8 \mathrm{~mm}$-AT. Following three days growth, two colony types are evident: large, AT-resistant and tiny, AT-sensitive clones. The tiny colonies are characteristic of the parental mutant hisO2355. Such clones are stably ATsensitive, giving rise to AT-resistant mutants only at the frequency characteristic of 


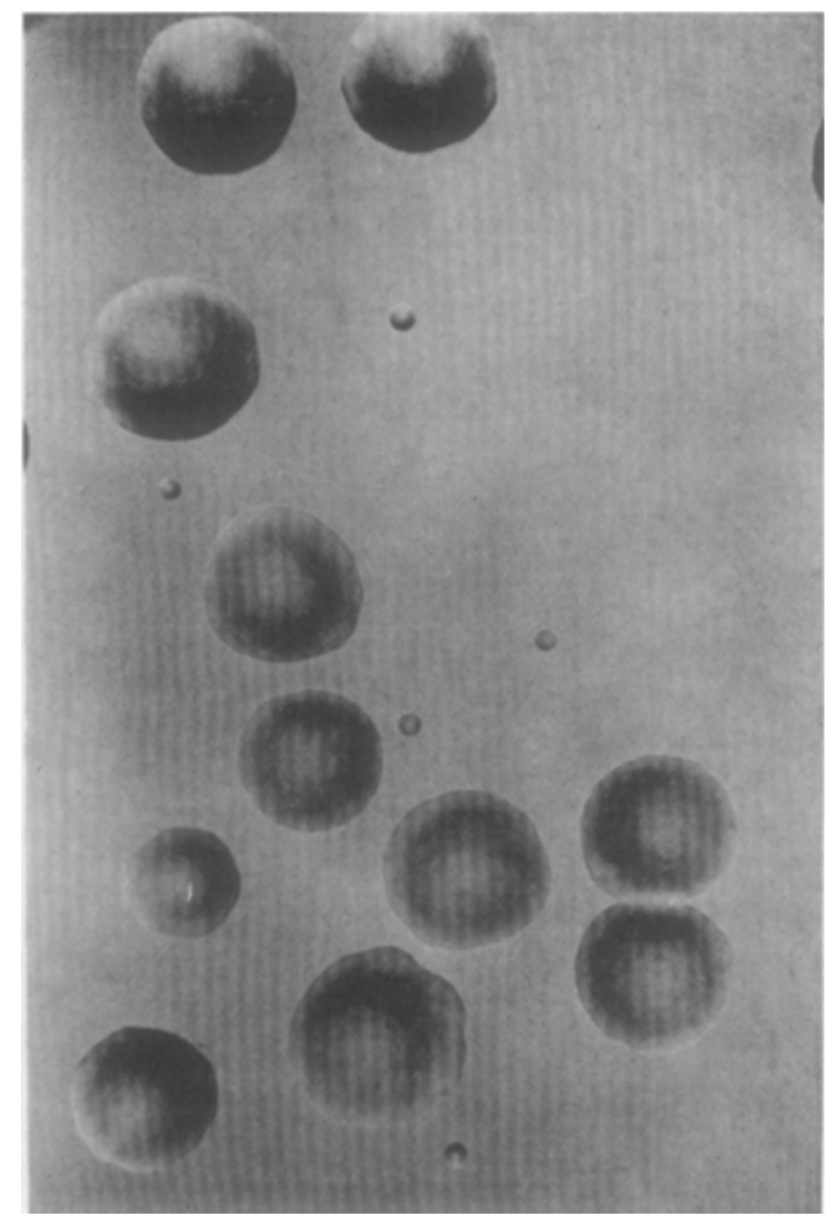

Fra. 1. Colony morphology of AT-resistant mutants and their AT-sensitive segregants.

hisO2355 (see section $(\mathrm{g})$, below). The small size of these clones demonstrates the sensitivity of haploid hisO2355 strains to AT inhibition. The large colonies in Figure I are those of the AT-resistant mutant. Despite repeated isolation and purification, these clones continue to segregate AT-sensitive progeny at high frequency. Thus, they are genetically unstable for their selected phenotype.

Instability is a standard feature of tandem genetic duplications (Campbell, 1963). The results presented below (sections (b) to (f)) indicate that these AT-resistant mutants harbor tandem duplications of chromosomal segments that include the his operon. In later sections ( $(\mathrm{g})$ to $(\mathrm{h})$ ), the formation mechanisms and adaptive significance of tandem duplication are considered.

\section{(b) Instability is dependent upon recombination}

Tandem genetic duplications generate haploid segregants as the result of homologous recombination between duplicated regions (Campbell, 1963). Tandem duplications are, therefore, stable in recombination-deficient backgrounds. The crosses shown in 
Figure 2 demonstrate that the instability of AT-resistant mutants is dependent upon recombination (recA function). A rec $A^{-}$mutation was introduced into AT-resistant mutants by use of the transductionally linked marker $\mathrm{srl}^{-}$. A $s r l^{-}$strain is unable to utilize D-sorbitol as a sole carbon source. As diagrammed in the first cross shown in Figure 2, a $s l^{+} r e c A^{-}$donor was used to transduce a number of independent $s r l^{-}$ rec $A^{+}$AT-resistant mutants, and the isogenic $\mathrm{Rec}^{+}$and $\mathrm{Rec}^{-}$recombinants were
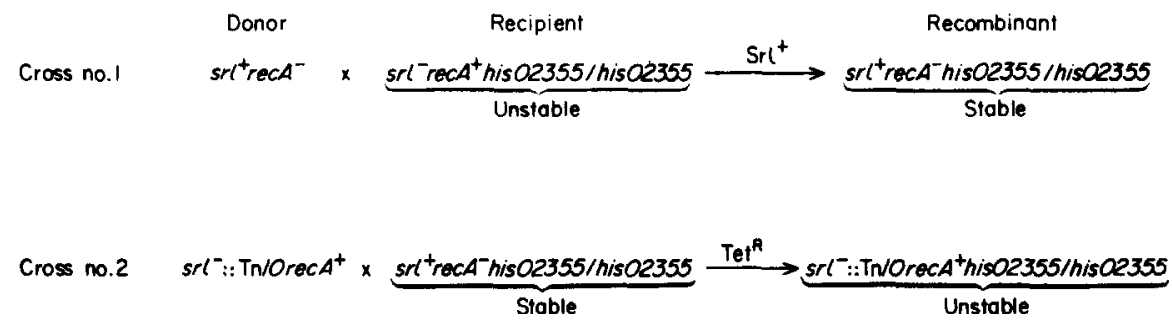

Fig. 2. Transductional crosses demonstrating that instability of AT-resistant mutants is dependent upon recombination. Selected phenotypes are indicated above the arrows. Donor strains used were TR2951 (his $\left.\triangle 63 \mathrm{srl}^{+} \operatorname{rec} A 1 \operatorname{str} A\right)$ and $\mathrm{TT520}\left(\operatorname{srl}-202: \operatorname{Tn} 10 \operatorname{rec} A^{+}\right)$.

identified among the $\mathrm{Srl}^{+}$progeny by their ultraviolet sensitivity. $\mathrm{Rec}^{+}$recombinants (10 were tested) exhibit the same high frequency of AT-sensitive segregants characteristic of AT-resistant mutants (average $=33 \%$ following 15 to 20 generations on non-selective growth for the 4 strains tested). $\mathrm{Rec}^{-}$recombinants (10 were tested) exhibit no segregation of AT-sensitive clones $(<0 \cdot 008 \%)$. The second cross shown in Figure 2 demonstrates that these stable strains still harbor the duplication. Donor strain TT520 (srl-202::Tn10) was used to transduce the Rec stable recombinants obtained from cross number one, selecting tetracycline resistance. Strain TT520 harbors an insertion of the translocatable tetracycline resistance determinant $\operatorname{Tn} 10$ (Kleckner et al., 1975) into the $s r l$ gene. Many transducing fragments that carry the srl ${ }^{-}:$:Tn10 region of the strain TT520 also carry rec $A^{+}$. Expression of such transducing fragments in infected cells yields $r e c A^{+}$function (at least transiently). Homologous recombination then proceeds to integrate $r e c A^{+}$-transducing fragment DNA into the recipient chromosome. In this manner, rec $A^{+}$alleles were re-introduced into the $\mathrm{Rec}^{-}$stable duplications; $r e c A^{+}$and $r e c A^{-}$recombinants were identified by their u.v.-sensitivity. When this was done, the instability characteristic of AT-resistant mutants reappeared in all of the six $\operatorname{rec} A^{+}$recombinants tested; none of $\operatorname{six} \operatorname{rec} A^{-}$ recombinants tested regained instability. From these experiments, we conclude that AT-resistant mutants are at least 4000 -fold more stable in rec $A^{-}$backgrounds. Thus, the genetic instability of AT-resistant mutants is dependent upon recombination.

\section{(c) Amino-triazole-resistant mutants have approximately twofold increased his enzyme levels}

If AT-resistant mutants harbor tandem duplications, their merodiploidy should be reflected hy increased levels of his hiosynthetic enzymes. A number of AT-resistant, mutants have been assayed for their content of histidinol dehydrogenase, the product of the his $D$ gene. Expression of $h i s D$ is under the exclusive control of the primary his promoter (Ely \& Ciesla, 1974). The level of this enzyme should reflect the gene dosage of hisD. Representative results of these measurements are presented in Table 2. 
Two independent AT-resistant mutants obtained from his02355 exhibit nearly twofold $(1 \cdot 7 \times)$ increased expression of hisD enzyme (lines 2, 3 and 4). The increase is less than twofold, probably because of haploid segregants among the diploid population at the time of assay. (Assays under repressed conditions require the addition of histidine to the growth media. Thus, haploid segregants are not counter-selected.) AT-sensitive segregants arising from AT-resistant mutants exhibit enzyme levels characteristic of haploid hisO23.55 (lines 5 and 6). The his $D$ enzyme levels exhibited by $1.8 \mathrm{~mm}$-AT-resistant mutants have never been observed to be greater than twice the level of hisO2355. We therefore feel that AT-resistant mutants isolated in this manner contain only two copies of the his operon.

(d) his 02355 merodiploids constructed by standard genetic procedures are amino-triazole-resistant

If AT-resistance can be caused by a tandem genetic duplication, then hisO235.j merodiploids constructed by more standard genetic procedures should also be AT-resistant. We have constructed such a merodiploid by mating donor strain TT2279

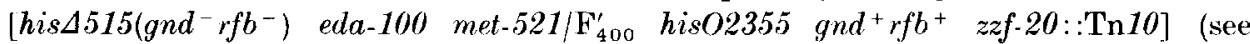
Materials and Methods for its construction) with recipient strain TR5369 (hisO235.5 rec $A 1$ ), selecting tetracycline-resistant conjugants. Donor strain TT2279 harbors an $\mathrm{F}^{\prime}$ his episome of Salmonella origin, into which the mutation his02355 and an insertion of the translocatable tetracycline-resistance determinant $\operatorname{Tn} 10 \quad(z z f-20: \operatorname{Tn} 10)$ have been transduced. The Tn 10 insertion present in this strain provides a selectable marker for episome transfer (tetracycline resistance) that is unrelated to the his operon. Exconjugants that arise from this mating (hisO2355 rec $11 / \mathrm{F}_{400}^{\prime}$ hisO2355 zzf-20::Tn 10 ) contain two copies of the hisO2355 operon. One such exconjugant (TT2280) was tested and found to be AT-resistant. We conclude, therefore, that the approximately twofold increase in gene dosage resulting from the presence of the $F^{\prime}$ episome is sufficient to confer AT-resistance to strains harboring mutation his 02355.

(e) Amino-triazole-resistant mutants are merodiploid for large chromosomal regions

The presence of duplicated DNA in AT-resistant mutants has been demonstrated genetically. The basic scheme for this has been to determine whether AT-resistant mutants can be made heterozygous for genetic markers near the histidine operon. If they can, then these strains must be merodiploid for those markers. To do this, we have isolated and characterized a large collection of auxotrophic and fermentationdefective mutants generated by insertion of the transposable tetracycline-resistance determinant $\operatorname{Tn} 10$. These mutations result from the linear insertion of the $\operatorname{Tn} 10$ element into defined structural genes (Kleckner et al., 1975).

Such mutations may be transduced selectively into recipient strains, because the Tn10 element itself specifies a selectable phenotype (tetracycline resistance) as well as causing the mutant phenotype. Selection for inheritance of $\operatorname{Tn} 10$ (by selecting tetracycline resistance) demands that recipient cells inherit the lesion caused by Tn10 insertion. Thus, when haploid strains inherit such Tn10 insertions, they also inherit the donor's auxotrophy or fermentation defect. If, however, the recipient strain is merodiploid for the Tn10 insertion site, tetracycline-resistant recombinants remain prototrophic. This is due to the presence of a second (wild-type) copy of the gene 
involved and the recessive nature of these mutations. Transductional crosses of this type allow rapid testing of whether a given strain is merodiploid for known $\operatorname{Tn} 10$ insertion sites.

The merodiploidy harbored by 77 independent AT-resistant mutants has been determined using this technique. Our method of isolating AT-resistant mutants guarantees them to be of independent origin (see Materials and Methods). The results of these tests are presented in Table 3. Each of 77 independent AT-resistant mutants is merodiploid for a contiguous array of chromosomal loci that includes the his operon. One minor and two major classes of duplications are revealed. Class I AT-resistant mutants (42 independent isolates) are merodiploid for the nearby loci pur $F$ and cys $A$. Isolates of this type are merodiploid for 8 to $17 \%$ of the Salmonella genome. Class III AT-resistant mutants are merodiploid for each of 13 loci tested extending fiom his through $l y s A$. Such strains are merodiploid for 18 to $26_{\%}^{\circ}$ of the chromosome. A single AT-resistant mutant (class II) is merodiploid for each of 10 loci extending from his through pheA.

None of the 77 AT-resistant mutants tested above was found to be merodiploid for the closest testable marker counter-clockwise from the his operon (tre $\left.e^{-}: \operatorname{Tn} 10\right)$. This raised the possibility that not all his genes are included in the duplicated material. Since the selection for AT resistance was thought to depend upon a gene dosage increase of $h i s B$ (the fourth structural gene distal to his $O$ ), the duplications might have been structured such that other his genes were not included in the duplicated material. This possibility has been eliminated by the following experiment.

Among our collection of is $^{-}:: \operatorname{Tn} 10$ insertion mutants are those in which the sites of Tn10 insertion (a strongly polar transcriptional block) within the his operon have been mapped (M. Johnston, unpublished data). Tn10 insertions have been identified in every his gene except hisE. Using the same rationale described above for testing chromosomal merodiploidy, these Tn 10 insertions may be used for testing the merodiploidy of individual his genes. In order to gain the most information from these crosses, it was necessary to first transduce the $\mathrm{his}^{-}:$:Trilo insertions into the hisO promoter-like mutant background. When these hisO2355 his ${ }^{-}:: \operatorname{Tn} 10$ strains are used as donors in crosses with AT-resistant recipients (selecting tetracycline resistance), the phenotypes of the recombinants yield information which confirms that (i) ATresistant mutants are merodiploid for all his genes and (ii) a doubling of his $B$ expression is the basis for AT-resistance. These conclusions are based upon the data pre-

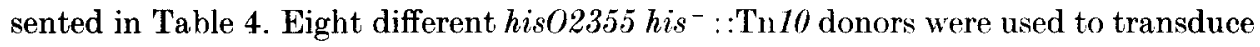
haploid and AT-resistant merodiploid recipients. selecting tetracycline resistance. The phenotypes of the resulting recombinants are presented in Table 4 . Whereas haploid recipients inherit the donor's histidine auxotrophy, AT-resistant recipients do not. Thus, AT-resistant mutants are merodiploid for all his genes tested (his $($-hisI). Furthermore, when the donor's Tn 10 insertion site is within or operator-proximal to his $B$, tetracycline-resistant recombinants are rendered AT-sensitive. (Strains with operator-proximal insertions are actually slightly more resistant to AT than haploid hisO2355, due to the presence of a low-level constitutive promoter located between the $h i s C$ and $h i s B$ genes (Atkins \& Loper, 1970); they are not however, fully ATresistant.) Insertions distal to his $B$ yield fully AT-resistant $\mathrm{His}^{+}$recombinants. Considering the strongly polar nature of Tn 10 insertions (Kleckner et al., 1975), these results confirm that the basis for duplication selection is an increase in his $B$ expression. The results also demonstrate that duplications include the entire his operon. 
(f) The large merodiploid state of amino-triazole-resistant mutants is transducible.

In strains harboring tandem genetic duplications. sequences that are widely separated in a normal chromosome are made contiguous at the junction between duplicated segments. Transduction of this novel sequence (often termed the "novel-joint"; Hershey, 1970) into normal (haploid) recipients promotes re-establishment of the donor's tandem duplication state in recipient cells. This is possible even when the amount of material included in the duplication is much too large to be carried by a single transducing fragment. Recombination events that account for this behavior are shown in Figure 3. A transducing fragment that carries the join-point of a tandem
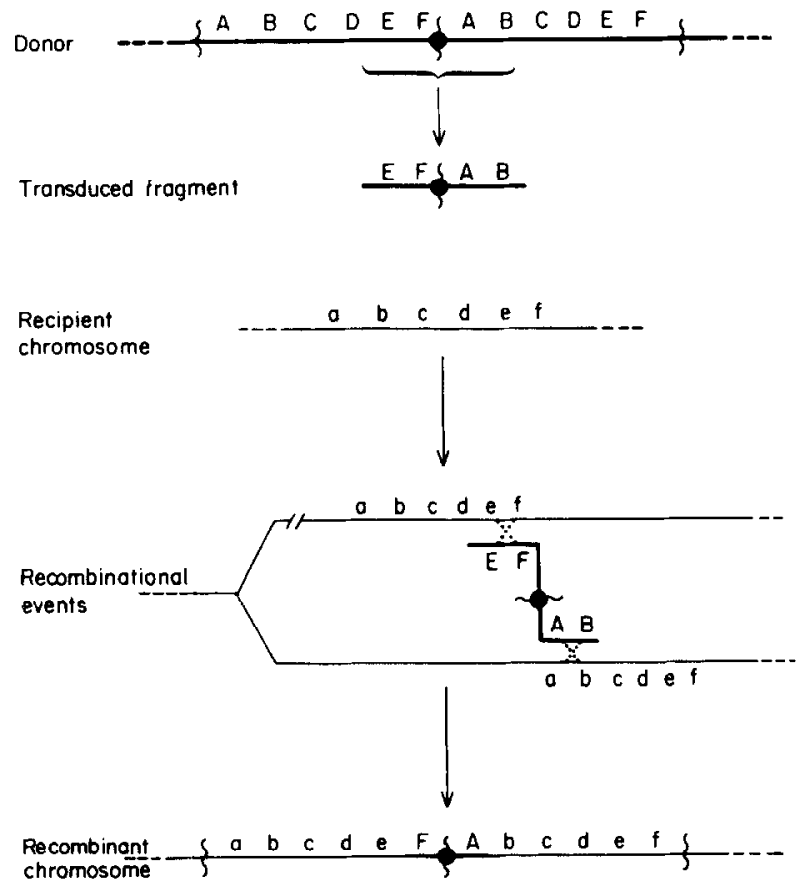

Fir. 3. A mechanism for transduetion of large tandem duplications (Campbell, 1963). Recipient. and donor DNAs are light and bold lined, respectively. Solid lines represent double-stranded DNA. Broken lines represent reciprocal recombination events.

duplication contains base-sequence homology to two widely separated regions of the recipient chromosome. When such a fragment enters a recipient cell, reciprocal recombination events between that fragment and two recipient chromosomes serve to regenerate the duplication state of the donor. In the resulting recombinant, most of the duplicated material is derived from recipient chromosomes; only material immediately adjacent to the join-point is derived from the donor. Thus, transduction of the merodiploid state of large tandem duplications may be detected, provided that a selectable donor marker and the join-point between duplicated donor segments are cotransducible. Transduction events such as those illustrated in Figure 3 were first suggested by Campbell (1965). Strong genetic evidence in support of these events has been subsequently presented (Hill et al., 1969; Anderson et al., 1976; Anderson \& Roth. 1978).

For AT-resistant mutants of the class III type. the chromosomal location of the 
duplication join-point and the ability of this join-point to promote merodiploid transductant formation may be demonstrated quite dramatically. Class IIl mutants are merodiploid for all loci tested in the region from his through $l y s A$ (see Table 3 ). They are not merodiploid for ser $A$ or more distal genes. lys $A$ and ser $A$ are separated by less than one minute on the linkage map of Salmonella (Sanderson \& Hartman, 1978). The duplication join-point in class III. AT-resistant mutants may be shown to be linked to the lysA gene by the following experiment: when a class III AT-resistant mutant is used as a transductional donor and a $l y s A^{-h}$ hoO2355 haploid strain is used as a recipient, a low but consistent fraction $(\sim 2 \%)$ of Lys ${ }^{+}$recombinants inherit the AT-resistant phenotype non-selectively. These data are presented in Table 5. The resulting Lys ${ }^{+}$AT-resistant recombinants are unstable for both their $\mathrm{Lys}^{+}$and AT-resistance phenotypes. They have thus inherited the large chromosomal merodiploidy characteristic of class III AT-resistant mutants. We presume that these? transductants arise by events outlined generally in Figure 3 and specifically in Figure 4. We interpret these results as indicating cotransduction between the $l y s A$ gene and the join-point of a tandem chromosomal duplication harbored by class III AT-resistant mutants. Linkage data of this type are strong evidence that a tandem chromosomal duplication is contained by AT-resistant mutants.
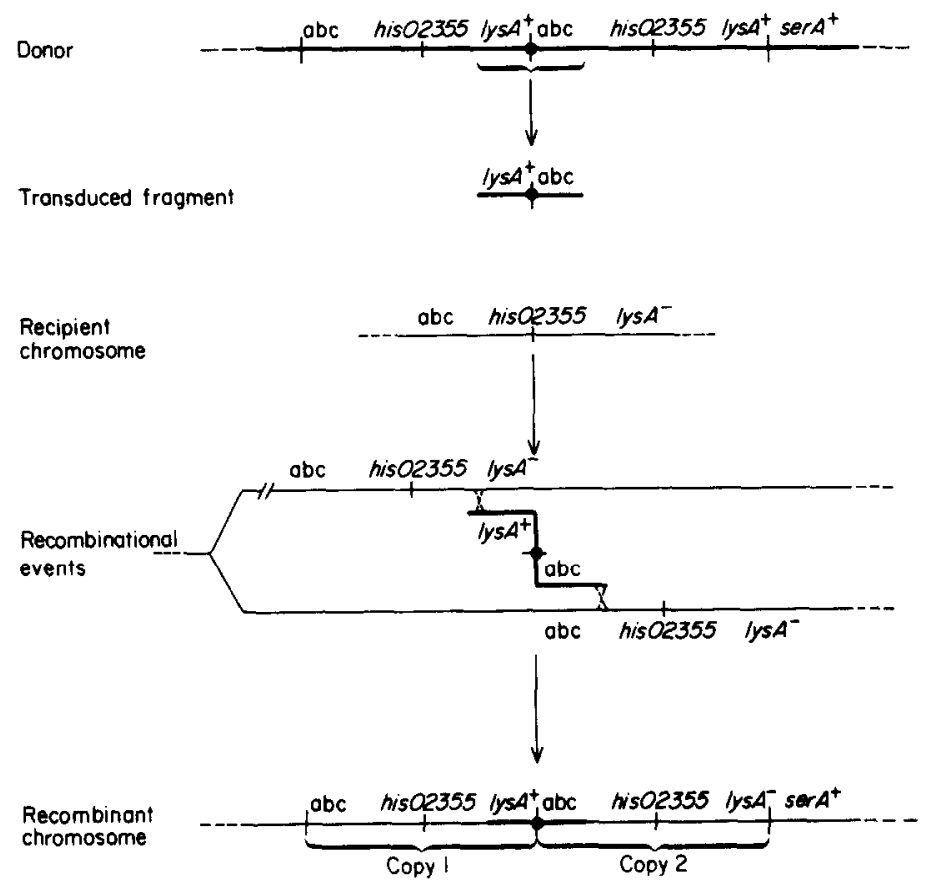

FIG. 4. Events involved in transducing the large merodiploidy harbored by class III A'I'-resistant mutants. Recipient and donor DNAs are light and bold-lined, respectively. Isower case letters abo are non-genetic indications of hypothetical base sequences.

\section{(g) Frequency of tandem duplications}

Tandem duplications of the his operon are frequent mutational events in Salmonella. Spontaneous AT-resistant mutants of strain TR5368 (hisO2355) occur at a frequency 
of $(6 \cdot 2 \pm 3 \cdot 1) \times 10^{-5}$ per cell (average \pm s.D. of 4 determinations). Of 238 AT-resistant mutants that have been purified and tested for stability 232 were found to be unstable and are therefore tandem duplications. The mechanism of formation of these duplications is highly dependent upon recombination. Spontaneous AT-resistant mutants of strain TR5369 (hisO2355 recA1) occur at a frequency of $(7 \cdot 3 \pm 4 \cdot 0) \times 10^{-7}$ per cell (average \pm S.D. of 4 determinations). This value is 85-fold less than the frequency for the isogenic (see Materials and Methods) rec $A^{+}$strain. Moreover, none of 76 independent AT-resistant mutants of $\mathrm{rec}^{-}$strain TR4255 (hi.sO235.5 srl-201 recA1 strA) harbors a tandem duplication of the his operon. When a rec $A^{+}$allele is transduced into these 76 AT-resistant mutants, none becomes unstable. The rec $A^{+}$derivatives of all 76 strains are haploid for the his operon, as evidenced by their His phenotypes upon inheritance of $\mathrm{his}^{-}: \mathrm{Tn} 10$ insertions. Thus. tandem duplications of the his operon are formed in a rec $A^{-}$background at a frequency of less than $10^{-8}$ per cell $\left(1 / 76 \times 7 \cdot 3 \times 10^{-7}\right)$. This is a 6000 -fold reduction of the frequency of tandem duplications seen in $\operatorname{rec} A^{+}$cells.

\section{(h) Amplification of the duplicated segment}

Tandem chromosomal duplications are inherently unstable structures. Normal recombination between the two copies of duplicated material lead either to loss of the duplication (Campbell, 1963; see section (a), above) or to triplication of the segment (Sturtevant, 1925; Parma et al., 1972). Cells harboring tandem triplications may be detected among cultures of AT-resistant duplications by virtue of an increased resistance to AT. When AT-resistant duplications are cultured under selective conditions (in media containing $1.8 \mathrm{~mm}-\mathrm{AT}$ ) for 20 to 40 generations, and samples are spread onto AT-containing plates, approximately 1 to $5 \%$ of the colonies are larger and more granular in appearance than those of duplication-containing strains. When purified and retested, the growth of these larger clones is consistently more resistant to A'T inhibition than is growth of duplication-containing strains. For the following reasons such clones are thought to harbor a tandem triplication of the chromosomal segment that is duplicated in their AT-resistant parents: (i) two types of segregants arise from these clones. One type is AT-sensitive (haploid). Another type has the colony morphology, AT-resistance levels, and instability characteristic of duplicationcontaining strains. (ii) Transduction of auxotrophic Tn10 insertions into these strains (see section (e), above) indicates that the chromosomal region carried in diploid (or greater) amount by these strains is the same as that carried by the parental duplication. (iii) Triploidy has been demonstrated genetically. We have in our stock collection his- mutations generated by insertion of the transposable kanamycin-resistance determinant Tn 5 (Berg et al., 1975), as well as those resulting from TnlO insertion. One such Tn5 insertion (isolated by D. Stetler) is within hisH. The more highly AT-resistant strains (triploids) were transduced with this donor (selecting kanamycin resistance), and the resulting recombinants are phenotypically $\mathrm{His}^{+} \mathrm{AT}^{\mathrm{R}} \mathrm{Kan}^{\mathrm{R}}$ (the site of $\operatorname{Tn} 5$ insertion is operator-distal to his $B$; thus recombinants remain highly AT-resistant). Such a recombinant was then used as a recipient for donor strain his $H^{-}:: \operatorname{Tn} 10$, selecting teracycline resistance (and kanamycin resistance). The resulting recombinants are phenotypically $\mathrm{His}^{+} \mathrm{AT}^{\mathrm{R}} \mathrm{Kan}^{\mathrm{R}} \mathrm{Tet}^{\mathrm{R}}$. Moreover, when these strains are grown non-selectively three types of haploid segregants are found: (a)

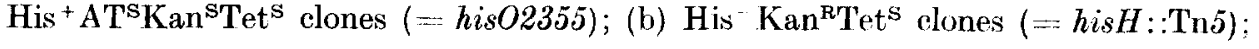




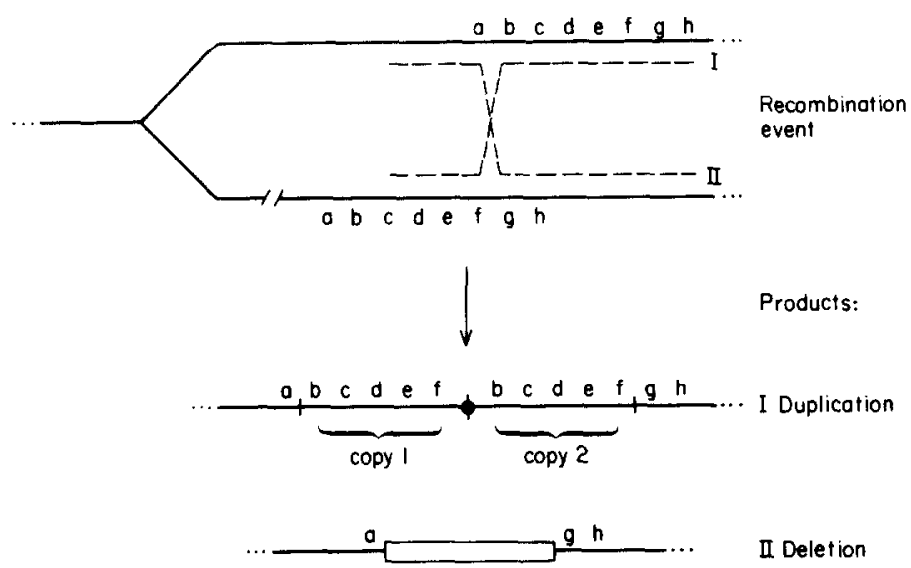

Frg. 5. Unequal crossing-over results in duplication (I) or deletion (I1) of the chromosomal segment bcdef. Solid lines indicate double-stranded DNA. Broken lines represent a reciprocal recombination event.

and (c) His ${ }^{-} \mathrm{Kan}^{\mathrm{S}} \mathrm{Tet}^{\mathrm{R}}$ clones $\left(=h i s H^{-}:: \mathrm{Tn} 10\right)$. The fact that these higher-level AT-resistant strains are phenotypically $\mathrm{His}^{+}$even after two different his $H$ insertions have been transduced into them confirms that they are genetically triploid for the his operon.

The frequency of triploids among cultures of duplication-containing strains is difficult to estimate. The growth advantage of triploids when compared to diploids in AT-containing media is slight. Thus, it is not possible to select triploids directly at higher AT concentrations. They are most easily identified by prolonged cultivation of duplication-containing strains under selective conditions. Under these conditions, triploids have a growth advantage and increase their fraction of the population. They may then be identified visually by their colony size and appearance on AT-containing plates. It may be demonstrated qualitatively that the mechanism of forming triploids from diploids depends upon recombination (as expected). When the stable rec $A^{-}$ AT-resistant duplications (see section (b), above) are cultivated selectively for 20 to 40 generations, no larger, more highly AT-resistant colonies are observed $(<0 \cdot 05 \%)$.

Although no direct proof has been obtained indicating that strains may be isolated which contain greater than triploid levels of amplification, we feel that continued selective cultivation of duplication and triplication-containing strains yields clones that harbor more tandem copies of the his operon. This observation is based upon the colony morphology and AT-resistance levels of strains that have been grown selectively for many generations.

\section{Discussion}

Strains harboring tandem duplications of chromosomal segments that include the histidine operon may be selected from populations of his $O$ promoter-like mutants. Such duplications exhibit resistance to the histidine analogue, 3-amino-1,2,4-triazole, because of a gene dosage effect upon expression of the his $B$ gene. These conclusions are based on a number of properties of AT-resistant mutants: (i) AT-resistant mutants are genetically unstable for their selected phenotype; such instability is dependent 
upon a functional recombination system. (ii) AT-resistant mutants express approximately twice the level of his enzymes as parental strains. (iii) Genetic tests indicate that AT-resistant mutants are merodiploid for large segments of the chromosome. (iv) For certain A'T-resistant mutants, a sequence that promotes AT-resistant merodiploid transductant formation (the join-point of a tandem duplication) may be cotransduced with a chromosomal marker distant to and unrelated to the his opsron.

The spontaneous frequency of tandem duplications that include the his operon is quite high $\left(6.2 \times 10^{-5}\right.$ per cell). Most such duplications are quite large. Two major classes of tandem his duplications are repeatedly isolated. One class is merodiploid for $\sim 13 \%$ of the genome, and another is duplicated for $\sim 22 \%$ of the chromosome. The fact that large tandem duplications cause no loss of function suggests that there may be few limits upon the maximum size of duplicated segments. This in turn, permits even widely separated homologous sequences to be available for the unequal exchanges (see below) that lead to duplications. It is likely that the high frequency and large size of duplications reflect these considerations.

The mechanism for formation of tandem his duplications is highly dependent upon recombination. Tandem duplications are formed in rec $A^{-}$backgrounds at least 6000 -fold less frequently than in $r e c A^{+}$backgrounds. We interpret this as indicating that most duplications are formed by normal (homologous) recombination between identical (or nearly identical) sequences located at different points on the chromosome. Such unequal crossing-over is depicted in Figure 5. Reciprocal recombination between the humologous sites $a-b$ and $f-g$ results in recombinant chromosomes (I) carrying a duplication of the sequence bcdef and (II) carrying a deletion of the same sequence. Considering the large size of his duplications, the deletion products would surely be lethal.

The origin and nature of the homologous sequences at different chromosomal sites remains to be determined. It is attractive to speculate that IS-sequences (Starlinger \& Saedler, 1972,1976) may be involved in the duplication process. Such sequences could provide the homology at separated chromosomal sites necessary for rec-dependent unequal crossing-over; they might also be involved in rec-independent exchanges since an IS sequence can promote illegitimate exchanges between one end of the IS and second points in the genome. Wild-type bacterial strains are thought to carry multiple copies of IS-sequences in their genomes (Saedler \& Heiss, 1973). The sites of such sequences are largely unknown, but some have been identified as points of F-factor insertion into the chromosome (Sharp et al, 1972; Ohtsubo et al., 1974; Davidson et al., 1975). It is interesting to note that both endpoints of the frequent class III duplications (one of which lies in the quite small lys A-serA interval) occur in regions of the Salmonella chromosome known to contain. Hfr points of origin (Sanderson et al., 1972). Ribosomal RNA genes have been shown to serve as regions of homology for duplication formation in E. coli (Hill et al., 1977). Genes for transfer RNAs might also provide homologous sequences.

The high frequency of tandem duplications and the ability to increase the number of copies of duplicated material suggest that bacteria might utilize this process as a form of gene amplification. The adaptive role that such amplification may play in population dynamics is apparent. Any selective conditions that favor cells containing two (or more) copies of a particular gene enrich a population in those cells harboring the appropriate tandem duplication. Continued selection and competition could lead 
to populations with higher levels of amplification. If growth conditions change so that a particular duplication is no longer favored, the cell population is not committed to its new genotype; the instability of duplications permits return to a predominantly haploid state. In a sense, this might be thought of as a rather erude regulatory mechanism that gains its specificity from natural selection. Although we know of no direct evidence demonstrating that such amplification is important to bacterial chromosomes its rolc in R-factor transitioning (Rownd \& Mickel, 1971) has been clearly demonstrated (Hashimoto \& Rownd, 1975). A more widespread use of this process for amplification of chromosomal genes by clonal selection would not seem surprising.

This work was supported by grants from the United States Public Health Service (GM-18663) and from the National Science Foundation (PCM76-15048). One of us (P.A.) was supported by a National Science Foundation Pre-doctoral Fellowship.

\section{RE'ERENUES}

Anderson, R. P. \& Roth, J. R. (1977). Annu. Rev. Microbiol. 31, 473-505.

Anderson, R. P. \& Roth, J. R. (1978). J. Mol. Biol. 119, 147-166.

Anderson, R. P., Miller, C. G. \& Roth, J. R. (1976). J. Mol. Biol. 105, $201-218$.

Atkins, J. F. \& Loper, J. C. (1970). Proc. Nat. Acad.Sci., U.S.A. 65, 925-932.

Berg, D., Davies, J., Allet, B. \& Rochaix, J. P. (1975). Proc. Nat. Acad. Sei., T.S.A. 72, 3628-3632.

Campbell, A. (1963). Virology, 20, 344-356.

Campbell, A. (1965). Virology, 27, 329-339.

Ciesla, Z., Sulvatore, F., Broach, J. R., Artz, S. W.\& Ames, B. N. (1975). Anal. Biochem. $63,44-55$.

Davidson, N., Deonier, R. C., Hu, S. \& Ohtsubo, E. (1975). Microbiology, 1, 56-71.

Ely, B. \& Ciesla, Z. (1974). J. Bacteriol. 120, 984-986.

Ely, B., Fankhauser, D. B. \& Hartman, P. E. (1974). Genetics, 78, 607-631.

Hashimoto, H. \& Rownd, R. H. (1975). J. Bacteriol. 123, 56-68.

Hegeman, G. D. \& Rosenberg, S. L. (1970). Annu. Rev. Miorobiol. 24, 429-462.

Hershey, A. D. (1970). Yearb. Carnegie Instn, 69, 717-722.

Hill, C. W. \& Combriato, G. (1973). Mol. Gen. Genet. 127, 197-214.

Hill, C. W., Schiffer, D. \& Berg, P. (1969). J. Bacteriol. 99, 274-278.

Hill, C. W., Grafstrom, R., Harnish, B. \& Hillman, B. (1977). J. Mol. Biol. 116, 407-408.

Hilton, J., Kearney, P.\& Ames, B. N. (1965). Arch. Biochem. Biophys. 112, 544-547.

Horiuchi, T., Horiuchi, S. \& Novick, A. (1963). Genetics, 48, 157-169.

Jacob, F., Ullman, A. \& Monod, J. (1964). C. R. H. Acad. Sci. 258, 3125-3128.

Kleckner, N., Chan, R. K., Tye, B. K. \& Botstein, D. (1975). J. Mol. Biol. 97, 561-575.

Langridge, J. (1969). Mol. Gen. Genet. 105, 74-83.

McEntee, K. (1976). Virology, 70, 221-222.

Miller, C. G. \& Roth, J. R. (1971).J. Mol. Biol. 59, 63-75.

Novick, A. \& Horiuchi, T. (1961). Cold Spring Harbor Symp. Quant. Biol. 26, 234-245.

Ohno, S. (1970). Evolution by Gene Duplication, pp. 160, Springer-Verlag, New York.

Ohtsubo, E., Deonier, R. C., Lee, H. J. \& Davidson, J. (1974). J. Mol. Biol. 89, 565-584.

Parma, D. H., Ingraham, L. J. \& Snyder, M. (1972). Genetics, 71, 319-335.

Peyru, G. \& Frankel, D. G. (1968). .J. Bacteriol. 95, 1273-1278.

Rigby, P. W. J., Burleigh, B. D. \& Hartley, B. S. (1974). Nature (London), 251, 200-204.

Roth, J. R. (1970). Meth. Enzymol. 17, 3-35.

Rownd, R. \& Mickel, S. (1971). Nature New Biol. 234, $40-43$.

Saedler, H. \& Heiss, B. (1973). Mol. Gen. Genet. 122, 267-277.

Sanderson, K. E. \& Hartman, P. E. (1978). Bacteriol. Rev. 42, 471-519.

Sanderson, D. E., Ross, H., Ziegler, L. \& Mäkelä, P. H. (1972). Bacteriol. Rev. 36, 608-637.

Scaife, J. \& Beckwith, J. (1966). Cold Spring Harbor Symp. Quant. Biol. 31, 403-408.

Schmieger, H. (1971). Mol. Gen. Genet. 110, 378-381. 
Sharp, P. A., Hsu, M.-T., Ohtsubo, E. \& Davidson, N. (1972). .J. Mol. Biol. 71, 471-497. Starlinger, P. \& Saedler, H. (1972). Biochimie, 54, 177-196.

Starlinger, P. \& Staedler, H. (1976). Curr. Top. Microbiol. Immunol. 75, 111-123.

Straus, D. S. (1974). Genetics, 78, 823-830.

Straus, D. S. \& Hoffmann, G. R. (1975). Genetics, 80, 227237.

Straus, D. S. \& Straus, L. D. (1976). J. Mol. Biol. 103, 143-154.

Sturtevant, A. H. (1925). Genetics, 10, 117-147.

Vogel, H. \& Bonner, D. (1956). J. Biol. Chem. 218. $97 \ldots 106$.

Voll, M. J. (1967). J. Mol. Biol. 30, 109-124.

Voll, M. .T. (1972). J. Bacteriol. 109, 741-750. 